\title{
Use of spermine and thiabendazole as analyte protectants to improve direct analysis of 16 carbamates \\ by gas chromatography-mass spectrometry in green vegetable matrices
}

\section{Cédric Przybylski • Véronique Bonnet}

Published online: 9 July 2009

(C) Springer-Verlag 2009

\section{Erratum to: Anal Bioanal Chem \\ DOI 10.1007/s00216-009-2773-1}

The authors have indicated that there is a mistake in Table 1 of this contribution. The molecular weights listed were incorrectly paired with the carbamate analyte listed for each. Please find the corrected table below.

The online version of the original article can be found at http://dx.doi. org/10.1007/s00216-009-2773-1.

C. Przybylski $(\bowtie)$

Laboratoire de Recherche et Développement Analytique,

Centre Technique de la Conservation des Produits Agricoles,

41 avenue Paul Claudel,

80480 Dury les Amiens, France

e-mail: cedric.przybylski@univ-evry.fr

V. Bonnet

Laboratoire des Glucides, UMR CNRS 6219,

Université de Picardie Jules Verne,

33 rue Saint Leu,

80039 Amiens, France

Present Address:

C. Przybylski

LAMBE, UMR CNRS 8587, Université d'Evry-Val d'Essonne,

Boulevard François Mitterrand,

91025 Evry, France 


\begin{tabular}{|c|c|c|c|c|c|}
\hline Name & MW (g/mol) & $\begin{array}{l}\text { Retention time in } \\
\text { full-scan mode (min) }\end{array}$ & $\begin{array}{l}\text { Start time period in } \\
\text { SIM mode (mid) }\end{array}$ & Ions $(\mathrm{m} / \mathrm{z})$ & $\begin{array}{l}\text { EU MRLs } \\
(\mathrm{mg} / \mathrm{kg})\end{array}$ \\
\hline ЕРTC & 189.12 & 6.41 & 6.00 & 190,164 & $0.1^{\mathrm{a}}, 0.05^{\mathrm{b}-\mathrm{c}}$ \\
\hline Propham & 179.09 & 7.50 & 7.00 & $\mathbf{1 4 4}, 115$ & $0.05^{\mathrm{a}-\mathrm{c}}$ \\
\hline Chlorpropham & 213.06 & 8.96 & 8.20 & $\mathbf{1 6 4}, 149$ & $0.05^{\mathrm{a}-\mathrm{c}}$ \\
\hline Carbofuran & 221.11 & 9.75 & 9.30 & 160,164 & $0.02^{\mathrm{a}-\mathrm{c}}$ \\
\hline Triallate & 303.00 & 10.50 & 10.10 & $\mathbf{1 2 7}, 171$ & $0.1^{\mathrm{a}-\mathrm{c}}$ \\
\hline Pirimicarb & 238.14 & 10.64 & & 225,267 & $1^{\mathrm{a}}, 5^{\mathrm{b}}, 2^{\mathrm{c}}$ \\
\hline Ethiofencarb & 225.08 & 10.81 & & $\mathbf{1 2 8}, 86$ & No value set \\
\hline Carbaryl & 201.08 & 11.47 & 11.15 & 107,168 & $0.05^{\mathrm{a}-\mathrm{c}}$ \\
\hline Prosulfocarb & 251.13 & 11.72 & & $\mathbf{2 5 5}, 186$ & $0.05^{\mathrm{a}-\mathrm{c}}$ \\
\hline Methiocarb & 225.08 & 11.92 & & $\mathbf{1 9 4}, 325$ & $0.2^{\mathrm{a}}, 1^{\mathrm{b}}, 0.1^{\mathrm{c}}$ \\
\hline Diethofencarb & 267.15 & 12.31 & & 160, 131 & $0.1^{\mathrm{a}}, 0.5^{\mathrm{b}}, 0.05^{\mathrm{c}}$ \\
\hline Mecarbam & 329.05 & 13.24 & 12.70 & 168,225 & $0.05^{\mathrm{a}-\mathrm{c}}$ \\
\hline Carbosulfan & 380.21 & 16.80 & 16.00 & 238, 167 & $0.05^{\mathrm{a}-\mathrm{c}}$ \\
\hline Fenoxycarb & 301.13 & 16.95 & & $\mathbf{9 3}, 137$ & $0.05^{\mathrm{a}-\mathrm{c}}$ \\
\hline Furathiocarb & 382.16 & 17.12 & & 251, 128 & $0.05^{\mathrm{a}-\mathrm{c}}$ \\
\hline Benfuracarb & 410.19 & 17.95 & & 268,143 & $0.05^{\mathrm{a}-\mathrm{c}}$ \\
\hline
\end{tabular}

